\title{
Delirium, a Symptom of UTI in the Elderly: Fact or Fable? A Systematic Review*
}

\author{
Seki A. Balogun, MBBS, and John T. Philbrick, MD \\ Division of General Medicine, Geriatrics/Palliative Care, University of Virginia Health System, Charlottesville, VA, USA
}

DOI:http://dx.doi.org/10.5770/cgj.17.90

\begin{abstract}
\section{Background}

In geriatrics, delirium is widely viewed as a consequence of and, therefore, a reason to initiate workup for urinary tract infection (UTI). There is a possibility that this association is overestimated. To determine the evidence behind this clinical practice, we undertook a systematic review of the literature linking delirium with UTI.
\end{abstract}

\section{Methods}

A MEDLINE search was conducted from 1966 through 2012 using the MESH terms "urinary tract infection" and "delirium", limited to humans, age 65 and older. The search identified 111 studies. Of these, five met our inclusion criteria of being primary studies that addressed the association of UTI and delirium. The studies were four cross-sectional observational studies and one case series. No randomized control trials were identified. All studies were published between 1988 and 2011. Four collected data retrospectively and one prospectively, with study sizes ranging from 14 to 1,285 . The methodological strength of the studies was evaluated using six standards adapted from a previous systematic review.

\section{Results}

Only two of the five studies adequately matched or statistically adjusted for differences in comparison groups. None of the studies evaluated subjects with equal intensity for the presence of delirium and UTI, nor did they have objective criteria for either diagnosis. In subjects with delirium, UTI rates ranged from $25.9 \%$ to $32 \%$ compared to $13 \%$ in those without delirium. In subjects with UTI, delirium rates ranged from $30 \%$ to $35 \%$, compared to $7.7 \%$ to $8 \%$ in those without UTI.

\footnotetext{
*Accepted for poster presentation at American Medical Director's Association (AMDA) Long Term Care Medicine, March 2013.
}

\section{Conclusions}

Few studies have examined the association between UTI and delirium. Though the studies examined conclude that there is an association between UTI and delirium, all of them had significant methodological flaws that likely led to biased results. Therefore, it is difficult to ascertain the degree to which urinary tract infections cause delirium. More research is needed to better define the role of UTI in delirium etiology.

Key words: delirium; urinary tract infection; older adults

\section{INTRODUCTION}

Delirium and urinary tract infections (UTI) are two very common conditions in the elderly. The incidence of both conditions increases with age and varies with different clinical sites. ${ }^{(1,2)}$ Urinary tract infections account for almost $25 \%$ of all infections in the elderly, particularly those in long term care facilities, ${ }^{(3)}$ while delirium is prevalent in up to $30 \%$ of elderly patients, with estimates being much higher postoperatively and in settings such as intensive care units. ${ }^{(4,5)}$ While delirium has multiple etiologies, it is widely viewed as one of the atypical symptoms of UTI in the elderly, ${ }^{(6,7)}$ and some physicians believe that the relationship between delirium and UTI is one of causation. ${ }^{(8,9)}$ They therefore initiate workup for urinary tract infection (UTI) whenever delirium occurs in a patient. However, there is the possibility that this association is overestimated, since there is also a high prevalence of asymptomatic bacteriuria in the elderly, particularly among those in nursing homes. Physicians who routinely search for a UTI in delirious patients will frequently find bacteriuria and treat the patient for a UTI, thinking that they have found the cause of the delirium. To determine the basis for this clinical practice, we undertook a systematic review of the literature linking delirium with UTI.

\section{METHODS}

A MEDLINE search was conducted from 1966 through October 2012 using the MESH terms "urinary tract infection" and 
"delirium", limited to humans, age 65 and older. References of relevant articles were also reviewed for papers not included in the initial search. The search identified 111 studies. Both authors reviewed these studies. Five studies met our inclusion criteria: all study designs except case reports; primary studies that address the association of UTI and delirium; enrollment of patients aged 65 years and older.

The studies were classified according to study type and the methodological strength was evaluated using six standards adapted from a previous systematic review. ${ }^{(10)}$ These standards addressed the assembly of study subjects and issues of bias in comparing groups and in the diagnosis of delirium and UTI. In particular, we were looking for study designs that limited "diagnostic suspicion bias" (knowledge of presence of an abnormal urine leading to documentation of delirium) and "exposure suspicion bias" (knowledge of presence of delirium leading to documentation of urine abnormalities). Because there were no randomized controlled trials, we did not include additional standards addressing issues of randomization and blinding. The standards are described below.

Standard 1: Adequate description of subject assembly process - To allow understanding of how assembly of study group could impact generalizability of study results, methods for subject selection should be clearly described such that the study could be replicated, identifying the same or a similar group of subjects. Total number of subjects eligible and actual number included in study should be stated, with reasons for exclusion.

Standard 2: Adequate description of subjects-To assist in generalizing study results, demographic and clinical information about the subjects should be presented, including summary information regarding age, gender, comorbidities, and clinical site of care.

Standard 3: Equality of comparison groups-To limit bias in studies with comparison groups, the groups should be matched for risk factors for delirium or UTI according to study design, either by matching or statistical adjustment.

Standard 4: Ascertainment of presence of disease and outcome performed equally in all study groups - To limit bias in the diagnosis of delirium and UTI, all subjects should have been evaluated with equal intensity for presence of (a) delirium and (b) UTI.

Standard 5: Clear criteria for diagnoses-To allow understanding of diagnostic certainty, objective criteria for the diagnosis of (a) delirium and (b) UTI must be provided.

Standard 6: Adequate description of treatment and subject follow-up-In studies in which patients are followed forward in time, to understand the interventions and their effects, all forms of treatment must be reported, as well as the results of follow-up testing for delirium. In addition, the numbers of patients lost to follow-up should be reported.

\section{RESULTS}

The five studies identified were comprised of one case series ${ }^{(8)}$ and four cross-sectional observational studies..$^{(11,12,13,14)}$ All were conducted in the United States except one, which was conducted in Scandinavia. ${ }^{(14)}$ No randomized control trials, case control, or cohort studies were found. The studies were published between 1988 and 2011 and used retrospective data collection; except for one cross-sectional study that collected data prospectively. ${ }^{(14)}$ The studies ranged in size from 14 to 1,285 subjects. Four of the studies were in hospital settings ${ }^{(8,11,12,13)}$ and one had community dwelling and institutionalized participants. ${ }^{(14)}$ Of those with delirium, UTI rates ranged from $25.9 \%$ to $32 \%$, compared to $13 \%$ in those without delirium. Of those with UTI, delirium rates ranged from $30 \%$ to $35 \%$, compared to $7.7 \%$ to $8 \%$ in those without UTI (Table 1 ).

There was general compliance with Standards 1 and 2 dealing with assembly and description of subjects. Only two of the five studies made any attempt to match or adjust comparison groups to make them comparable (Standard 3). None of the studies fully complied with Standards 4 and 5, which required that delirium and UTI be sought with equal diligence in all subjects, and that there be clear criteria for the diagnosis of delirium and UTI.

\section{DISCUSSION}

Our systematic review has found that few studies have directly examined the association between UTI and delirium. Since no randomized controlled trials have evaluated this association, it is impossible to determine the degree to which urinary tract infection causes delirium and how successful treatment of UTI could lead to improvement in symptoms of delirium. Though the five studies in our review report an association between delirium in elderly patients and UTI, all have significant methodological flaws with potential for bias.

First, most of the studies examined did not provide adequate statistical adjustment for risk factors of delirium or UTI in comparison groups (Standard 3). ${ }^{(8,12,13)}$ The study that best adjusted for risk factors in the comparison group $^{(14)}$ showed only a small association (OR 1.9; 95\% CI 1.1-1.3) between UTI and delirium, the smallest association of the five studies. Inadequate statistical adjustment in the other studies is likely to have led to a biased estimation of the association.

Second, the lack of objective diagnostic criteria for delirium and UTI in studies also presents the potential for bias (Standard 5), since most of the studies use chart review and discharge ICD-9 codes for these two conditions, making verification of the accuracy of these diagnoses difficult to do.

Finally, in the reviewed studies, the door to diagnostic and exposure suspicion biases (Standard 4) was wide open, likely leading to overestimation of the association between 
BALOGUN: DELIRIUM AND UTI IN THE ELDERLY

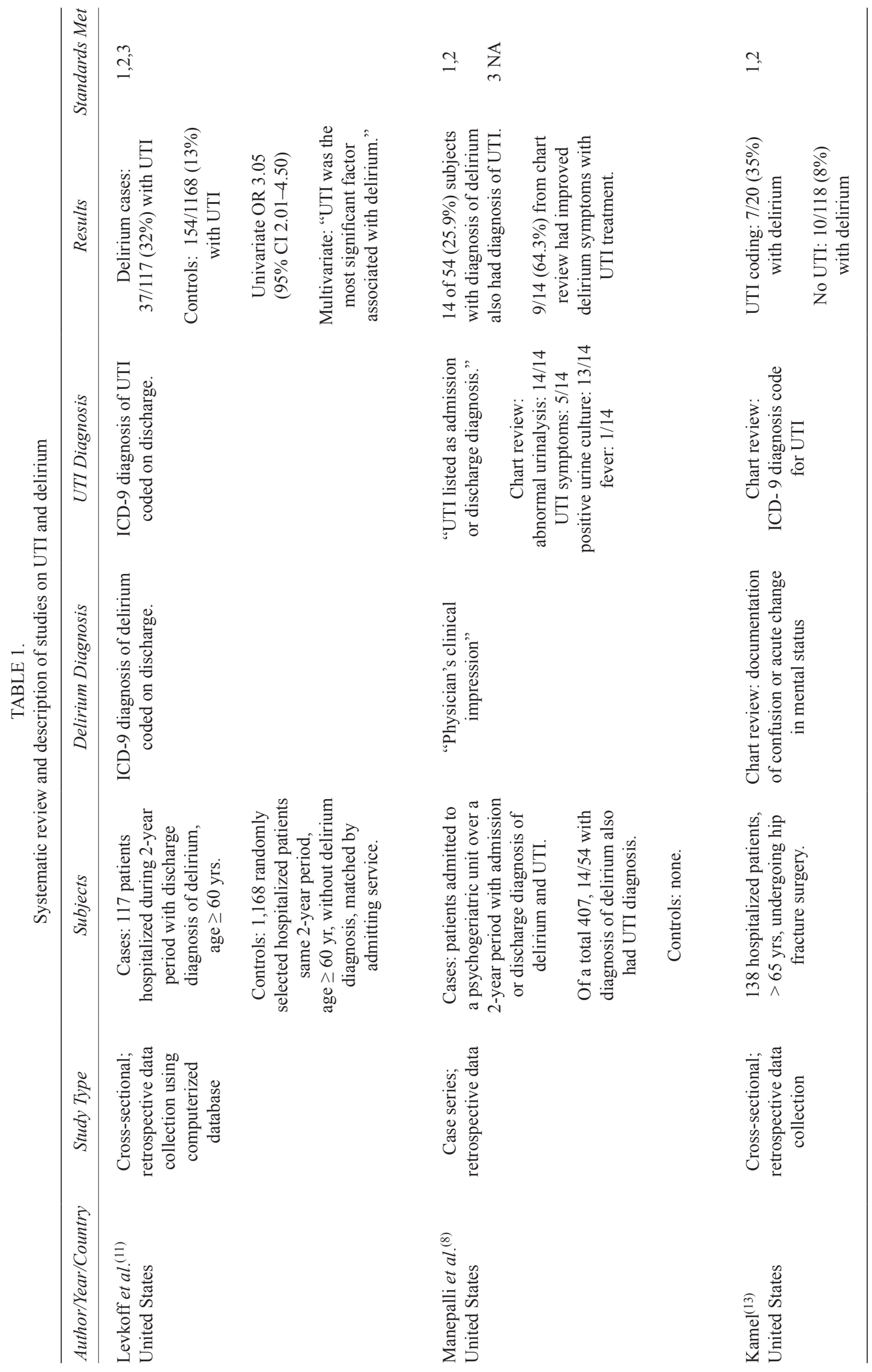

CANADIAN GERIATRICS JOURNAL, VOLUME 17, ISSUE 1, MARCH 2014 
BALOGUN: DELIRIUM AND UTI INTHE ELDERLY

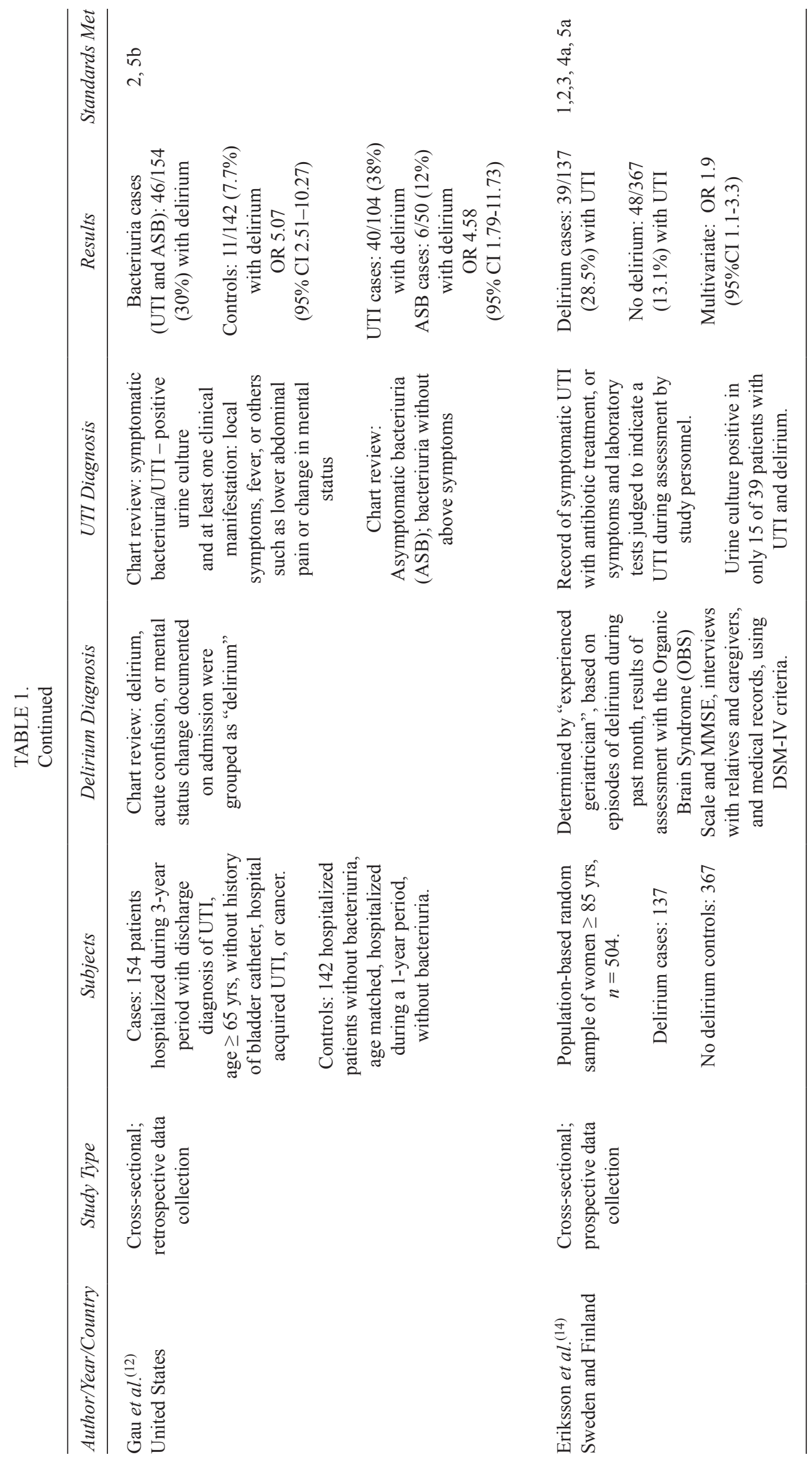


UTI and delirium. Given the widespread belief in the relationship of delirium and UTI, if a physician admits a patient with delirium, the presence of UTI would be sought and documented in discharge diagnoses; or, if a physician admits a patient with UTI, presence of delirium would be sought and documented in discharge diagnoses. The only way to definitively avoid these two key biases in a study would be to examine with equal diligence all patients for both delirium and UTI and to record all of the results.

An argument for causation based on these studies is difficult to make. The cross-sectional study designs make it impossible to determine what came first, UTI or delirium. Therefore, the important temporal relationship between the two conditions was not explored in these studies. The strength of association between UTI and delirium is modest at best. There is little specificity for UTI as the cause of delirium since there are multiple other causes. The study by Eriksson et al., ${ }^{(14)}$ the least prone to bias, on multivariate analysis had the lowest odds ratio among the reviewed studies for the UTIdelirium association, and found other risk factors (presence of Alzheimer's disease, multi-infarct dementia, depression, heart failure) with larger odds ratios.

Overall, it is reasonable to conclude that there is an association between delirium and sufficiently symptomatic UTI, just as there is for other conditions such as presence of dementia, ${ }^{(8,14)}$ depression, ${ }^{(8,14)}$ heart failure, ${ }^{(14)}$ chronic kidney disease, ${ }^{(11)}$ use of psychotropic medications or multiple medications, ${ }^{(8)}$ and advanced age. ${ }^{(8,15)}$

On the other hand, it is also reasonable to conclude that asymptomatic bacteriuria-without dysuria, frequency, bladder discomfort, or fever-is unlikely to cause a patient to become delirious, as reported in the study by Gau et al., ${ }^{(12)}$ and that factors other than an abnormal urinalysis play a more dominant role in the development of delirium.

We recommend that, in evaluating elderly patients with delirium, all clinically plausible etiologies be considered, including a change of environment such as hospitalization, especially in those with baseline cognitive impairment. A reflexive examination of the urine in delirious elderly patients, with an end of the search for explanations if the urine is abnormal, is simply not sufficient and, based on our review, has no sound scientific justification. However, when a physician is faced with a positive urine culture in a patient without UTI symptoms and a negative workup for other causes for delirium, it still may make clinical sense to treat the infection in the hope of clearing the delirium.

It is also critically important for physicians to keep in mind the potential risks of overuse of antibiotics in elderly patients, particularly the danger of clostridium difficile infection. ${ }^{(16)}$ Unfortunately this practice is still quite common. For example, a recent emergency room study showed that almost half of older patients prescribed antibiotics for UTI had subsequent negative urine cultures. ${ }^{(17)}$

Overall, further research to better define the role of UTI in the etiology and management of delirium in the elderly is warranted.

\section{CONFLICT OF INTEREST DISCLOSURES}

The authors declare that no conflicts of interest exist.

\section{REFERENCES}

1. Calijouw AA, den Elzen PJ, Cools JM, et al. Predictive factors of urinary tract infections among the oldest old in the general population. A population-based prospective follow up study. BMC Medicine. 2011;9:57.

2. Nicolle LE. Urinary tract infections in the elderly. Clin Geriatr Med. 2009;25(3):423-36.

3. Foxman B. Epidemiology of urinary tract infections: incidence, morbidity and economic costs. Am J Med. 2002;113(1 Suppl):5S-13S.

4. Inouye SK. Delirium in hospitalized older patients: recognition and risk factors. J Geriatr Psychiatry Neurol. 1998;11:118-25.

5. Burock JM. Delirium in the elderly. Med Health $R I$. 2012;95(7):214-19.

6. Eriksson I, Gustafson Y, Fagerstrom L, et al. Prevalence and factors associated with urinary tract infections (UTIs) in very old women. Arch Gerontol Geriatr. 2010;50(2):132-35.

7. Mccue JD. Urinary tract infections in the elderly. Pharmacotherapy. 1993;13(2P2):515-35.

8. Manepalli J, Grossberg GT, Mueller C. Prevalence of delirium and urinary tract infection in a psychogeriatric unit. J Geriatr Psychiatry Neurol. 1990;3(4):198-202.

9. Lipowski ZJ. Delirium due to infection. In: Lipowski ZJ, editor. Delirium: acute brain failure in Man. Springfield, IL: Charles C Thomas; 1980. p.409-25.

10. Philbrick JT, Shumate R, Siadaty MS, et al. Air travel and venous thromboembolism: a systematic review. J General Intern Med. 2007;22:107-14.

11. Levkoff SE, Safran C, Cleary PD, et al. Identification of factors associated with the diagnosis of delirium in elderly hospitalized patients. J Am Geriatr Soc. 1988;36(12):1099-104.

12. Gau JT, Shibeshi MR, Lu IJ, et al. Interexpert agreement on diagnosis of bacteruria and urinary tract infection in hospitalized older adults. J Am Osteopath Assoc. 2009;109(4):220-26.

13. Kamel HK. The frequency and factors linked to a urinary tract infection coding in patients undergoing hip fracture surgery. J Am Med Dir Assoc. 2005;6(5):316-20.

14. Eriksson I, Gustafson Y, Fagerstrom L, et al. Urinary tract infection in very old women is associated with delirium. Int Psychogeriatrics. 2011;23(3):496-502.

15. Lipowski SJ. Update on delirium. Psychiatr Clin North Am. 1992;15(2):335-46.

16. Johnson S. Recurrent Clostridium difficile infection: a review of risk factors, treatments, and outcomes. $J$ Infect. 2009;58(6):403-10.

17. Gordon LB, Waxman MJ, Ragsdale L, et al. Overtreatment of presumed urinary tract infection in older women presenting to the emergency department. J Am Geriatr Soc. 2013;61(5):788-92.

Correspondence to: Seki A. Balogun, MBBS, Division of General Medicine, Geriatrics/Palliative Care, University of Virginia Health System, P.O. Box 800901, Charlottesville, VA 22908, USA

E-mail: sab2s@virginia.edu 